\title{
THE SDSS-IV EXTENDED BARYON OSCILLATION SPECTROSCOPIC SURVEY: LUMINOUS RED GALAXY TARGET SELECTION
}

\author{
Abhishek Prakash ${ }^{1}$, Timothy C. Licquia ${ }^{1}$, Jeffrey A. Newman ${ }^{1}$, Ashley J. Ross ${ }^{2,3}$, Adam D. Myers ${ }^{4}$, Kyle S. Dawson ${ }^{5}$, \\ Jean-Paul Kneib $^{6,7}$, Will J. Percival ${ }^{3}$, Julian E. Bautista ${ }^{5}$, Johan Comparat ${ }^{8}$, Jeremy L. Tinker ${ }^{9}$, David J. Schlegel ${ }^{10}$, \\ Rita Tojeiro $^{11}$, Shirley Ho ${ }^{12}$, Dustin Lang ${ }^{12}$, Sandhya M. RaO ${ }^{1}$, Cameron K. McBride ${ }^{13}$, Guangtun Ben Zhu ${ }^{14}$, \\ Joel R. Brownstein ${ }^{5}$, Stephen Bailey ${ }^{10}$, Adam S. Bolton ${ }^{5}$, Timothée Delubac ${ }^{6}$, Vivek Mariappan ${ }^{5}$, \\ Michael R. Blanton ${ }^{9}$, Beth Reid ${ }^{15}$, Donald P. Schneider ${ }^{16}$, Hee-Jong Seo ${ }^{17}$, Aurelio Carnero Rosell ${ }^{18,19}$, \\ AND FRANCISCO PRADA $8,20,21$ \\ ${ }^{1}$ PITT PACC, Department of Physics and Astronomy, University of Pittsburgh, Pittsburgh, PA 15260, USA; abp15@ pitt.edu \\ ${ }^{2}$ Center for Cosmology and Astro-Particle Physics, Ohio State University, Columbus, OH 43210, USA \\ ${ }^{3}$ Institute of Cosmology \& Gravitation, Dennis Sciama Building, University of Portsmouth, Portsmouth, PO1 3FX, UK \\ ${ }_{5}^{4}$ Department of Physics and Astronomy, University of Wyoming, Laramie, WY 82071, USA \\ ${ }^{5}$ Department of Physics and Astronomy, University of Utah, Salt Lake City, UT 84112, USA \\ ${ }^{6}$ Laboratoire d'Astrophysique, Ecole Polytechnique Fédérale de Lausanne Observatoire de Sauverny, 1290 Versoix, Switzerland \\ 7 Aix Marseille Université, CNRS, LAM (Laboratoire d'Astrophysique de Marseille) UMR 7326, F-13388, Marseille, France \\ ${ }^{8}$ Instituto de Física Teórica, (UAM/CSIC), Universidad Autónoma de Madrid, Cantoblanco, E-28049 Madrid, Spain \\ ${ }^{9}$ Center for Cosmology and Particle Physics, Department of Physics, New York University, 4 Washington Place, New York, NY 10003, USA \\ ${ }^{10}$ Lawrence Berkeley National Laboratory, One Cyclotron Road, Berkeley, CA 94720, USA \\ ${ }^{11}$ School of Physics and Astronomy, St Andrews, KY16 9SS, UK \\ 12 Bruce and Astrid McWilliams Center for Cosmology, Department of Physics, Carnegie Mellon University, 5000 Forbes Avenue, Pittsburgh, PA 15213, USA \\ ${ }^{13}$ Harvard-Smithsonian Center for Astrophysics, Harvard University, 60 Garden Street, Cambridge, MA 02138, USA \\ ${ }^{14}$ Department of Physics and Astronomy, Johns Hopkins University, Baltimore, MD 21218, USA \\ ${ }^{15}$ Berkeley Center for Cosmological Physics, LBL and Department of Physics, University of California, Berkeley, CA 94720, USA \\ ${ }^{16}$ Department of Astronomy and Astrophysics, 525 Davey Laboratory, The Pennsylvania State University, University Park, PA 16802, USA \\ ${ }_{17}$ Department of Physics and Astronomy, Ohio University, Athens, OH 45701, USA \\ ${ }^{18}$ Observatório Nacional, Rua Gal. José Cristino 77, Rio de Janeiro, RJ-20921-400, Brazil \\ ${ }^{19}$ Laboratório Interinstitucional de e-Astronomia,-LIneA, Rua Gal. José Cristino 77, Rio de Janeiro, RJ-20921-400, Brazil \\ ${ }^{20}$ Campus of International Excellence UAM+CSIC, Cantoblanco, E-28049 Madrid, Spain \\ ${ }^{21}$ Instituto de Astrofísica de Andalucía (CSIC), Glorieta de la Astronomía, E-18080 Granada, Spain \\ Received 2015 August 18; accepted 2016 April 11; published 2016 June 8
}

\begin{abstract}
We describe the algorithm used to select the luminous red galaxy (LRG) sample for the extended Baryon Oscillation Spectroscopic Survey (eBOSS) of the Sloan Digital Sky Survey IV (SDSS-IV) using photometric data from both the SDSS and the Wide-field Infrared Survey Explorer. LRG targets are required to meet a set of color selection criteria and have $z$-band and $i$-band MODEL magnitudes $z<19.95$ and $19.9<i<21.8$, respectively. Our algorithm selects roughly 50 LRG targets per square degree, the great majority of which lie in the redshift range $0.6<z<1.0$ (median redshift 0.71 ). We demonstrate that our methods are highly effective at eliminating stellar contamination and lower-redshift galaxies. We perform a number of tests using spectroscopic data from SDSS-III/ BOSS ancillary programs to determine the redshift reliability of our target selection and its ability to meet the science requirements of eBOSS. The SDSS spectra are of high enough signal-to-noise ratio that at least $89 \%$ of the target sample yields secure redshift measurements. We also present tests of the uniformity and homogeneity of the sample, demonstrating that it should be clean enough for studies of the large-scale structure of the universe at higher redshifts than SDSS-III/BOSS LRGs reached.
\end{abstract}

Key words: catalogs - cosmology: observations - galaxies: distances and redshifts - galaxies: general galaxies: photometry - methods: data analysis

\section{INTRODUCTION}

Many studies have found that massive galaxies, and in particular red, elliptical galaxies, tend to reside in massive dark matter halos and cluster strongly (e.g., Postman \& Geller 1984; Kauffmann et al. 2004). The most luminous galaxies in clusters and groups populate a narrow range of color and intrinsic luminosity (Postman \& Lauer 1995). These galaxies, which constitute the most massive, the most luminous, and the reddest (in rest-frame color) of all galaxies are typically referred to as "luminous red galaxies" (LRGs). Given both their bright intrinsic luminosities (allowing them to be studied to higher redshifts than typical $L^{*}$ galaxies) and their strong clustering, LRGs are excellent tracers of the large-scale structure of the universe.
LRGs have been previously used to study large-scale structure by a variety of investigations, most notably the Sloan Digital Sky Survey (SDSS; York et al. 2000) and the SDSSIII/Baryon Oscillation Spectroscopic Survey (BOSS), as well as the 2dF-SDSS LRG and QSO survey (e.g., Eisenstein et al. 2001; Cannon et al. 2006). In combination, SDSS-I, SDSS-II, and SDSS-III targeted LRGs at $z \lesssim 0.7$ to a magnitude limit of $i<19.9$ and $i_{\text {fiber2 }}<21.5$ (Eisenstein et al. 2001, 2005, 2011; Dawson et al. 2013). The methods used to select LRGs for these studies are limited in redshift range as a result of using optical photometry alone for selection. Identifying LRGs with shallow optical photometry becomes prohibitively difficult at higher redshifts as the $4000 \AA$ 
break passes into the near-infrared and colors overlap strongly with $\mathrm{M}$ stars.

New multi-wavelength imaging is now available which allows high-redshift LRGs to be selected much more efficiently than optical-only imaging would make possible. In particular, optical-infrared (optical-IR) colors provide a powerful diagnostic for separating galaxies and stars (Prakash et al. 2015), as well as a diagnostic of redshift. As a result, infrared observations from satellites such as the Wide-field Infrared Survey Explorer (WISE; Wright et al. 2010) provide additional information for targeting LRGs in regions of optical color space that would otherwise be heavily contaminated by stars.

Increasing our current sample of LRGs to higher redshifts will allow measurements of the baryon acoustic oscillation (BAO) feature, and hence of the expansion rate of the universe (Lin \& Mohr 2003; Seo \& Eisenstein 2003; Ross et al. 2008), during the era when accelerated expansion began. An optical + WISE selection makes it possible to target LRGs in the redshift range $0.6 \lesssim z \lesssim 1$ efficiently (Prakash et al. 2015); with spectroscopy of these targets, we can obtain stronger constraints on the BAO scale at these redshifts. At even higher redshift, other tracers such as quasi-stellar objects (QSOs, quasars) and emission line galaxies (ELGs) can be used to provide further complementary probes of the BAO scale. In combination, these target classes can provide powerful constraints on the evolution of cosmic acceleration across a wide range of redshifts. This led to the conception of a new survey, the extended Baryon Oscillation Spectroscopic Survey (eBOSS; Dawson et al. 2015) as part of SDSS-IV (M. Blanton et al. 2016, in preparation).

The LRG component of eBOSS will obtain spectra for $\sim 375,000$ objects. Approximately 265,000 of these are expected to be LRGs in the redshift range $0.6<z<1.0$, with a median redshift of $z \sim 0.7$. The main goal of this spectroscopic campaign is to produce more precise measurements of the BAO signal at $0.6<z<1.0$, thus extending probes of the BAO scale using LRGs beyond the BOSS redshift range. eBOSS LRGs are also expected to yield a $4 \%$ measurement of redshift space distortions (RSD) which will allow improved tests of general relativity at these redshifts (e.g., Beutler et al. 2014a, 2014b; Samushia et al. 2014).

Altogether, SDSS-IV/eBOSS will produce a spectroscopic sample of both galaxies and quasars over a volume that is ten times larger than the final SDSS-III BOSS sample, although at lower target density. This sample will enable a wide range of scientific studies beyond a BAO measurement. For example, the resulting sample of hundreds of thousands of LRGs extending to $z=1$ will be useful for a variety of studies of the evolution of the brightest elliptical galaxies, including measurements of luminosity functions, mass functions, size evolution, and galaxy-galaxy lensing.

In this paper, we describe the algorithm used to select LRG targets for the eBOSS survey. Further technical details about eBOSS can be found in companion papers on quasar selection (Myers et al. 2015), ELG selection (Comparat et al. 2015), survey strategy (Dawson et al. 2016), and the Tractor analysis of WISE data (Lang et al. 2014).

The paper is organized as follows. In Section 2 we outline the goals of eBOSS and the requirements placed on the LRG sample to meet these goals. The parent imaging data used for eBOSS LRG target selection is outlined in Section 3. In Section 4 we describe our new method of LRG selection and supporting tests for this method that were conducted during BOSS. In Section 5 we describe the eBOSS LRG targeting algorithms and the meaning of the relevant targeting bits, while Section 6 uses the latest results from eBOSS to test the target selection algorithm. An important criterion for any large-scale structure survey is sufficient homogeneity to facilitate modeling of the distribution of the tracer population, i.e., the "mask" of the survey. In Section 7, we use the full eBOSS target sample to characterize the homogeneity of eBOSS LRGs. We present conclusions and future implications for eBOSS LRGs in Section 8.

Unless stated otherwise, all magnitudes and fluxes in this paper are corrected for extinction using the dust maps of Schlegel et al. (1998), hereafter SFD, and are expressed in the AB system (Oke \& Gunn 1983). The SDSS photometry has been demonstrated to have colors that are within 3\% of being on an AB system Schlafly \& Finkbeiner (2011). We use a standard $\Lambda \mathrm{CDM}$ cosmology with $H_{0}=100 h \mathrm{~km} \mathrm{~s}^{-1} \mathrm{Mpc}^{-1}$, $h=0.7, \Omega_{\mathrm{M}}=0.3$, and $\Omega_{\Lambda}=0.7$, which is broadly consistent with the recent results from Planck (Planck Collaboration et al. 2014).

\section{COSMOLOGICAL GOALS OF EBOSS AND IMPLICATIONS FOR LRG TARGET SELECTION}

\subsection{Overall Goals for the LRG Sample}

The primary scientific goals of the eBOSS LRG survey are to constrain the scale of the BAO to $1 \%$ accuracy over the redshift regime $0.6<z<1.0$. This requires selecting a statistically uniform set of galaxies with the desired physical properties for which spectroscopic redshifts can be efficiently measured. The density of selected LRGs must not strongly correlate with either tracers of potential imaging systematics (e.g., variations in the depth of the imaging) or with astrophysical systematics such as Galactic extinction and stellar density.

\subsection{Target Requirements for LRGs}

As explained in Dawson et al. (2016), a density of $50 \mathrm{deg}^{-2}$ spectroscopic fibers are allocated to eBOSS LRGs and a density of $40 \mathrm{deg}^{-2}$ LRGs with redshifts $0.6<z<1.0$ is required, over the projected $7500 \mathrm{deg}^{-2}$ survey footprint, to meet the eBOSS scientific goals (see Dawson et al. 2016 for more details). If one were to consider eBOSS in isolation, this corresponds to a requirement that $80 \%$ of eBOSS LRG targets result in a spectroscopically confirmed galaxy with $0.6<z<1.0$. However, given that BOSS observed a density of $12 \mathrm{deg}^{-2}$ LRGs (assuming half of the BOSS galaxies with $z>0.6$ are LRGs, consistent with Ross et al. 2014), eBOSS can obtain its required number density of LRGs if it observes $28 \mathrm{deg}^{-2}$ additional LRGs with $0.6<z<1.0$. Additionally, we require the redshifts be accurate to better than $300 \mathrm{~km} \mathrm{~s}^{-1} \mathrm{rms}$ and robust such that the fraction of catastrophic redshift errors (exceeding $1000 \mathrm{~km} \mathrm{~s}^{-1}$ ) is $<1 \%$ in cases where the redshifts are believed to be secure. The construction of a sample designed to fulfill these requirements is described in Sections 4 and 5 .

A further requirement to obtain robust $\mathrm{BAO}$ measurements is that the density of selected LRGs must not strongly correlate with either tracers of potential imaging systematics (e.g., variations in the depth of the imaging) or with astrophysical systematics such as Galactic extinction and stellar density. 
BOSS has shown that fluctuations associated with surveys artifacts can be handled effectively via weighting schemes provided the amplitude of fluctuations is relatively small (Ross et al. 2012). To facilitate weighting schemes in future clustering studies, we require that that fluctuations in the expected target density as a function of potential imaging systematics, stellar density, and Galactic extinction be less than $15 \%$ (total variation around mean density). We require density differences due to imaging zero-point variations in any single band to be below $15 \%$ as well. Tests of the homogeneity of the LRG target sample are presented in Section 7.

\section{PARENT IMAGING FOR TARGET SELECTION}

\subsection{Updated Calibrations of SDSS Imaging}

All eBOSS LRG targets rely on imaging from the SDSS-I/ II/III. SDSS photometry was obtained by the SDSS telescope (Gunn et al. 2006) using its wide-field imaging camera (Gunn et al. 1998) in the ugriz system (Fukugita et al. 1996). SDSS-I/ II primarily obtained imaging over the $\sim 8400 \mathrm{deg}^{2}$ "Legacy" area, $~ 90 \%$ of which was in the North Galactic Cap (NGC). This imaging was released as part of SDSS Data Release 7 (DR7; Abazajian et al. 2009). The legacy imaging area of the SDSS was expanded by $2500 \mathrm{deg}^{2}$ in the South Galactic Cap (SGC) as part of DR8 (Aihara et al. 2011). The SDSS-III/ BOSS survey used this DR8 imaging for target selection over $\sim 7600 \mathrm{deg}^{2}$ in the NGC and $\sim 3200 \mathrm{deg}^{2}$ in the SGC (Dawson et al. 2013). LRG targets for eBOSS have been selected over the same footprint covered by BOSS; however, ultimately eBOSS will obtain spectroscopy for LRGs over a roughly $7500 \mathrm{deg}^{2}$ subset of this BOSS area, utilizing $50 \%$ of the dark time for six years in SDSS-IV. In this available time, it would not be possible to observe the full extragalactic footprint available from SDSS imaging.

Although conducted over the same area as BOSS, eBOSS target selection takes advantage of updated calibrations of the SDSS imaging. Schlafly et al. (2012) have applied the "ubercalibration" technique of Padmanabhan et al. (2008) to imaging from the Pan-STARRS survey (Kaiser et al. 2010), achieving an improved global calibration compared to SDSS DR8. The improvements in the photometric accuracy are very modest compared to DR8, typically less than $0.5 \%$. More importantly, BOSS was still acquiring imaging during its program, and therefore there was no single photometric solution for the full footprint. In contrast, eBOSS is targeting from a single photometric solution. Targeting for eBOSS is conducted using SDSS imaging that is calibrated using the Schlafly et al. (2012) Pan-STARRS solution. We will refer to this as the "updated" photometry below.

Specifically, targets are selected using the updated SDSS photometry stored in the calib_obj files, the basic imaging catalog files used in the SDSS-III data model. ${ }^{22}$ The updated Pan-STARRS-calibrated photometry will be made available as part of a future SDSS Data Release. The magnitudes provided in these files are Pogson magnitudes (Jones 1968) rather than the asinh magnitudes used for some SDSS data releases (Lupton et al. 1999). We use model magnitudes for all colors and fluxes used in selection. The model magnitudes are obtained by first determining what type of model (exponential or de Vaucouleurs) best fits the object image in

\footnotetext{
${ }^{22}$ For example, http://data.sdss3.org/datamodel/files/PHOTO_SWEEP/ RERUN/calibObj.html.
}

the "canonical" band (typically $r$, but other bands may be used if they have a higher signal-to-noise ratio $(\mathrm{S} / \mathrm{N})$ ), and then using the model fit from the canonical band (convolved with the appropriate point-spread function (PSF)) to obtain fluxes in each filter. Additionally, we also apply flux limits based upon an object's fiber 2 mag values; i.e., the total flux within a $2^{\prime \prime}$ diameter of the object center, corresponding to the aperture of a BOSS spectroscopic fiber (Smee et al. 2013), after convolving the imaging data to achieve a standard $2^{\prime \prime}$ seeing.

\subsection{WISE}

The eBOSS LRG target selection algorithm also relies on infrared photometry from WISE (Wright et al. 2010). WISE observed the full sky in four infrared channels centered at 3.4, 4.6, 12, and $22 \mu \mathrm{m}$, which we refer to as $W 1, W 2, W 3$, and $W 4$, respectively. For eBOSS LRGs, we use the W1-band only. WISE magnitudes are commonly measured in the Vega system, but we convert to the $\mathrm{AB}$ system for LRG selection. ${ }^{23}$ Over the course of its primary mission and the "NEOWISE post-cryo" continuation, WISE completed two full scans of the sky in the $W 1$ - and W2-bands. Over $99 \%$ of the sky has 23 or more exposures in $W 1$ and $W 2$, and the median coverage is 33 exposures. We use the "unWISE" forced photometry from Lang et al. (2014), which photometered custom coadds of the WISE imaging at the positions of all SDSS primary sources. Using forced photometry allows accurate flux measurements to be obtained even for significantly blended sources, including objects below the significance threshold for WISE-only detections. Since the WISE W1 PSF is relatively broad (6.1 arcsec FWHM, 4 times larger than typical SDSS seeing), many sources are blended and forced photometry presents substantial advantages. Additionally, forced photometry allows us to leverage the relatively deep SDSS photometry to measure fluxes of WISE sources that are otherwise below the detection threshold. The same canonical morphological model is used in fitting photometry of the optical SDSS and infrared WISE images, therefore consistently measuring colors across all bands. Using unWISE photometry instead of the Wright et al. (2010) WISE catalog increases the size of the resulting eBOSS LRG sample by $\sim 10 \%$.

\section{SELECTION OF HIGH- $z$ LRGS}

Our overall goal is to cleanly select a sample of LRGs at redshifts beyond 0.6. In this redshift regime, however, optical photometry alone becomes insufficient for discriminating these high- $z$ objects from foreground stars in our galaxy because both LRGs and red stars occupy the same region in optical colorcolor space. It is also not feasible to separate stars from galaxies reliably based on morphological information, as the $\mathrm{S} / \mathrm{N}$ of SDSS photometry for these objects is low. Prakash et al. (2015) presented a new technique which eliminates almost all stellar contamination by combining both optical and infrared imaging data and applying a simple cut in optical-IR color-color space. This takes advantage of the prominent $1.6 \mu \mathrm{m}$ "bump" in the spectral energy distributions of LRGs and other objects with old stellar populations (John 1988), which results from the minimum in the opacity of $\mathrm{H}^{-}$ions. The lowest wavelength channel of the WISE satellite is centered at $3.4 \mu \mathrm{m}$, almost

${ }^{23} W 1_{\mathrm{AB}}=W 1_{\mathrm{Vega}}+2.699$. 


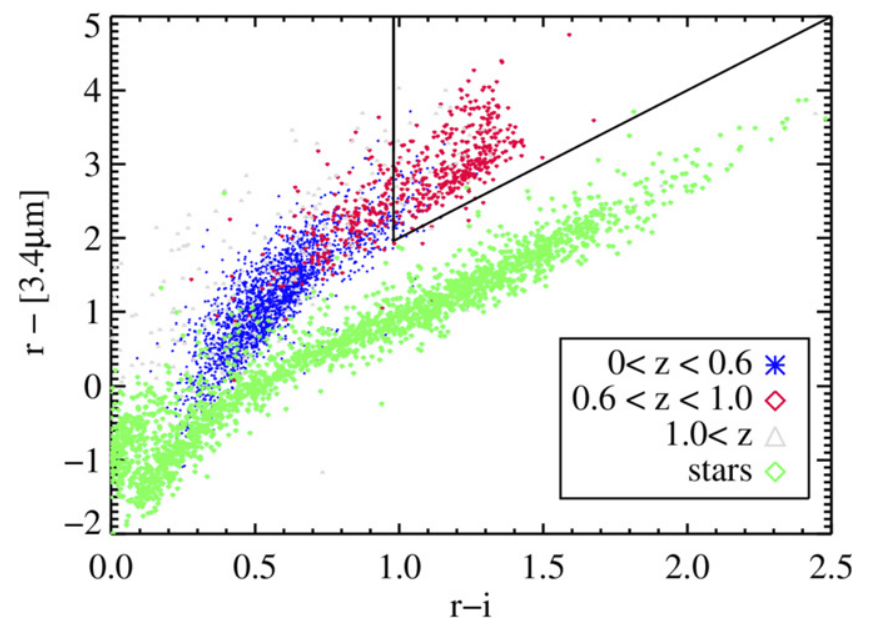

Figure 1. Optical-infrared color-color plot for galaxies observed by WISE and CFHTLS with photometric redshifts from the COSMOS survey. Blue symbols represent galaxies with photometric redshifts of $z<0.6$, red diamonds represent galaxies at $0.6<z<1.0$, and cyan triangles represent galaxies at $z>1.0$. Stars are represented by green diamonds. The triangular area depicts the broad selection presented in Equations (1) and (2). Photometric redshifts are taken from the COSMOS photo- $z$ catalog of Ilbert et al. (2008) and optical photometry is from the catalog of Gwyn (2011), transformed to SDSS passbands. The conversion relation can be found at the CFHTLS webpage (http://www.cadc-ccda.hia-iha.nrc-cnrc.gc.ca/en/megapipe/docs/filt.html).

perfectly in sync with the bump at $z \sim 1$. In that paper, the authors presented a variety of optimization tests based on CFHTLS photometry, DEEP2 spectroscopic redshifts, and COSMOS photometric redshifts. This method has been adapted here to meet eBOSS requirements specifically.

Figure 1 shows both stars and galaxies in a plot of $r-W 1$ verses $r-i$ color, where $W 1$ indicates the magnitude of a source in the WISE $3.4 \mu \mathrm{m}$ passband (on the AB system) and $r$ and $i$ indicate SDSS model magnitudes in the appropriate passband. Stars separate increasingly from the galaxy population in near-IR-optical color space as redshift increases, allowing clean discrimination of galaxies at $z>0.6$ from stars. Simultaneously, $r-i$ color increases with increasing redshift (particularly for intrinsically red galaxies) as the $4000 \AA$ A break shifts redward, allowing a selection specifically for higher-redshift objects. While the combination of optical and IR imaging provides an excellent means of removing stellar contamination from an LRG target sample, this approach also means that we are limited to objects that are detected by both SDSS and WISE. The detections are performed on the optical SDSS images only. WISE-only detections have not been utilized in the eBOSS target catalogs.

As a basic color selection for characterizing potential eBOSS LRG targets, we select all objects that satisfy the criteria

$$
\begin{gathered}
r-i>0.98, \text { and } \\
r-W 1>2.0 \times(r-i),
\end{gathered}
$$

where all magnitudes are corrected for Galactic extinction. These cuts were determined by examining the location of objects of known redshift and rest-frame color in color-color space, as in Figure 1. The clear separation seen between the locuses of stars and galaxies in Figure 1 is not so clear when a similar plot is made using SDSS photometry because of the latter's lower $\mathrm{S} / \mathrm{N}$; i.e., the gap between the two populations is partially filled in due to objects with noisy measurements. The selection cuts above were optimized by assessing a figure of merit which is a linear combination of fraction LRGs and low-redshift galaxies selected by a given color selection and normalized by the total number of selected objects (see Equation (9) and Figure 11 of Prakash et al. 2015). Hence, we are primarily optimizing for the purity of the sample, rather than its completeness. Further details on the motivation for this selection and various tests on optimization can be found in Prakash et al. (2015).

To test this new selection technique, we targeted 10,000 objects satisfying this selection in a BOSS ancillary program in 2012-2013 (see the appendix of Alam et al. 2015). Selection was limited to objects with $z_{\text {Model }}<20 ; 98 \%$ of the spectra yielded secure redshift measurements. These redshift estimates were found to be reproducible when observed multiple times. An additional 5000 LRGs were selected by relaxing the $r-i$ color requirement to $r-i>0.85$ in order to estimate the number of LRGs missed by the color cuts in Equation (1). The distributions of observed colors as a function of redshift for the resulting sample of 15,000 LRGs is presented in Figure 2.

Our method of combining optical and infrared photometry for this selection is unique; however, the specific choice of color cuts is not. We are able to cleanly select similar samples of LRGs by using different color combinations; e.g., $r-W 1$ and $r-z$, or $i-W 1$ and $i-z$. As can be seen in Figure 2, incorporating multiple colors can improve the efficiency of identifying true LRGs in the redshift range of interest by rejecting lower-redshift objects. We tested two parallel selection algorithms with different color selections in another BOSS ancillary program, Sloan Extended Quasar, ELG and LRG Survey (SEQUELS), to select the algorithm which is best poised to meet eBOSS requirements, as described in Appendix B.1. The purity of the eBOSS sample (i.e., the level of contamination by stars and low- $z$ galaxies) will be discussed further in Section 6.

\section{THE EBOSS LRG TARGET SELECTION ALGORITHM}

In this section, we describe in detail the final selection algorithm for SDSS-IV/eBOSS LRGs. At the high redshifts of the LRGs $(z>0.6)$, the $4000 \AA$ break moves into the SDSS $i$ band. As some objects are too faint to be detected by SDSS imaging in the $r$-band, flux measurements can occasionally be negative; by making color cuts in flux space rather than magnitude space, this poses no problems. However, for convenience, we describe the selection algorithm and flux limits in terms of extinction-corrected $\mathrm{AB}$ magnitudes and colors here.

To summarize our selection methods: we first employ photometric processing flags to eliminate those objects with problematic imaging. ${ }^{24}$ To ensure robust selection while maintaining a sufficient $\mathrm{S} / \mathrm{N}$ in eBOSS spectra, we also apply a variety of flux limits. Finally, to maximize the fraction of targets that are in fact high-redshift LRGs, we apply several color cuts. In the following sub-sections, we detail all the selections used for creating the eBOSS LRG sample.

\subsection{Photometric Flags for the LRG Sample}

Since many of the SDSS imaging runs overlap on the sky, an object may be observed twice or more (Stoughton et al. 2002).

\footnotetext{
${ }^{24}$ https://www.sdss3.org/dr8/algorithms/photo_flags_recommend.php
} 


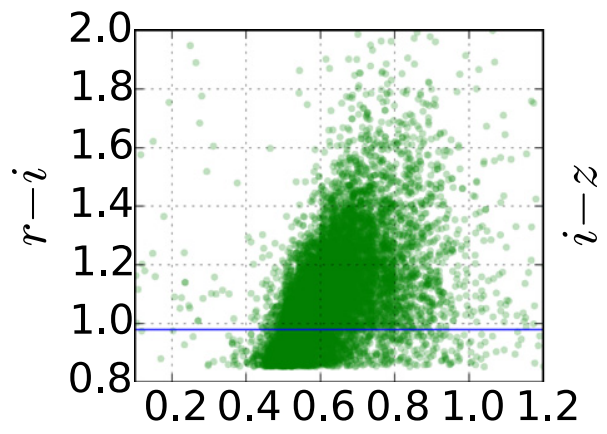

$z$

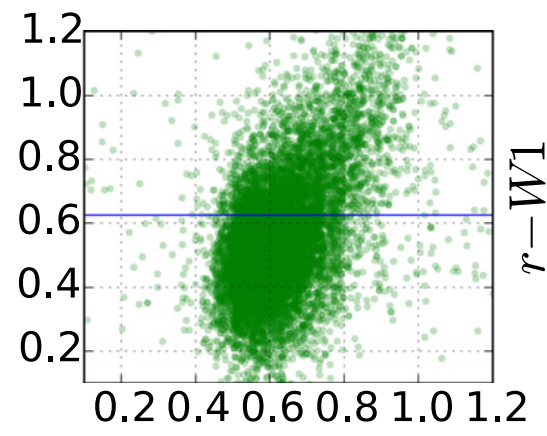

z

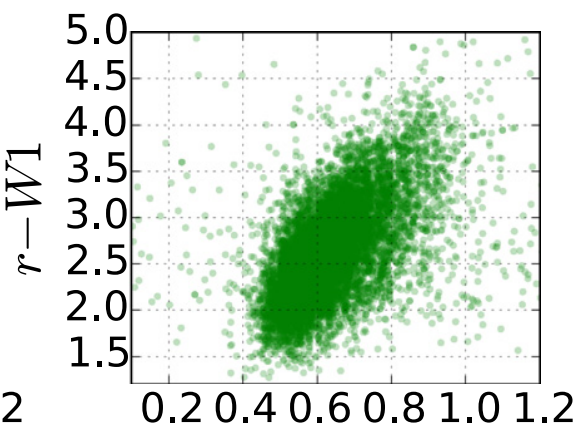

$z$

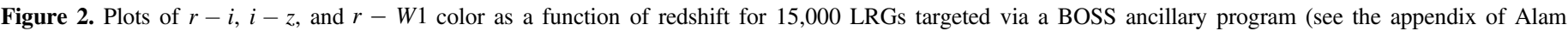

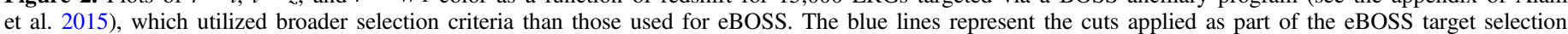

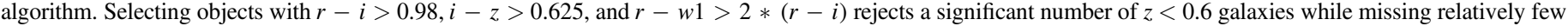
$z>0.6$ LRGs.

Only one observation is designated as the primary observation of the object during the resolve process. Hence, to exclude duplicate objects we enforce the following logical condition on the RESOLVE_STATUS bit-mask:

$$
\text { (Resolve_status \& Survey_primary) } \neq 0 \text {. }
$$

\subsection{Magnitude Limits}

The median $5 \sigma$ depth for photometric observations of point sources in the SDSS is $u=22.15, g=23.13, r=22.70$, $i=22.20$, and $z=20.71$ (Dawson et al. 2016). Additionally, we require a detection of the flux in the $W 1$ forced photometry for an object to be targeted. Keeping these requirements in mind, we apply the following flux limits to the entire sample:

$$
\begin{gathered}
\text { MODEL_IV } \mathrm{AR}_{r, i, z} \neq 0, \\
z_{\text {Fiber } 2} \leqslant 21.7, \\
19.9 \leqslant i_{\text {Model }} \leqslant 21.8, \\
z_{\text {Model }} \leqslant 19.95, \\
W 1_{\text {vega }} \neq 0, \text { and } \\
W 1_{\mathrm{AB}} \leqslant 20.299,
\end{gathered}
$$

where MODEL_IVAR are the inverse variances on the model fluxes in $r$-, $i$-, and $z$-bands. Equation (4) implies that errors in flux measurement are physically meaningful. The application of Equation (5) serves to maintain a sufficiently high $\mathrm{S} / \mathrm{N}$ of the eBOSS spectra. This cut is similar in spirit to the $i_{\text {Fiber2 }}$ cut that was used for the BOSS CMASS galaxy sample (Eisenstein et al. 2011). We apply the lower limit defined in Equation (6) in order to avoid targeting $i<19.9$ BOSS CMASS galaxies, which generally lie at lower redshifts and have been observed previously. $W 1_{\text {vega }}$ being nonzero implies that the photometry is reliable, while Equation (9) ensures that WISE flux measurements have a $\mathrm{S} / \mathrm{N}$ greater than 5 (Wright et al. 2010). The $i$ and $z$ faint magnitude limits are set to achieve the required target density of $\sim 60$ targets $\mathrm{deg}^{-2}$ matching the eBOSS fiber allocation for LRGs (Dawson et al. 2015), while maximizing the brightness of targets. LRGs are given lower priority for selection than the other main target class, QSOs, and hence a non-negligible fraction cannot be targeted due to fiber collisions. As a result, we must select 60 targets per square degree to end up with 50 LRG targets per square degree placed on fibers.

\subsection{Color Selection}

We use the $r-W 1$ (optical-IR) color for separating LRGs from stars. ${ }^{25}$ The optical colors of galaxies are used to ensure that the targeted objects are intrinsically red and lie in the desired redshift range. We thus apply the following three selection criteria:

$$
\begin{gathered}
r-i>0.98, \\
r-W 1>2.0 \times(r-i), \text { and } \\
i-z>0.625 .
\end{gathered}
$$

Equations (10) and (11) represent the basic LRG color selection discussed at the beginning of Section 4 and are identical to Equations (1) and (2), the color cuts used in initial tests of LRG selection. We use Equation (12) to reduce contamination from $z<0.6$ galaxies.

The overall eBOSS LRG selection algorithm is shown schematically as a flow chart in Figure 3. The details of this algorithm were optimized based upon a pilot survey, the SEQUELS, which is summarized in the appendix of Alam et al. (2015); the SEQUELS LRG selection algorithm is detailed in Appendix B.1.

In addition to the LRGs targeted by Equations (3)-(12), we target a small number of objects, 200 over the $10,000 \mathrm{deg}^{2}$ SDSS imaging area, via a different but related algorithm. These objects have $i_{\text {Model }} \geqslant 21.8$ and are designated LRG_IDROP. These are not significant for BAO studies but constitute a separate sample designed to identify rare objects at extremely high redshifts. Further details are provided in an appendix to this paper (see Appendix A).

\section{TESTS OF THE TARGET SELECTION ALGORITHM}

In this section, we assess the results of our target selection methods using the current eBOSS data. We use the automated spectral classification, redshift determination, and parameter measurement pipelines of SDSS-III BOSS which are described in Bolton et al. (2012), to reduce and analyze spectra of eBOSS targets. To assess the true redshifts of LRG sample, we have

\footnotetext{
${ }^{25}$ Note that we do not explicitly use any morphological cuts, but rather separate stars and galaxies based only on their colors.
} 


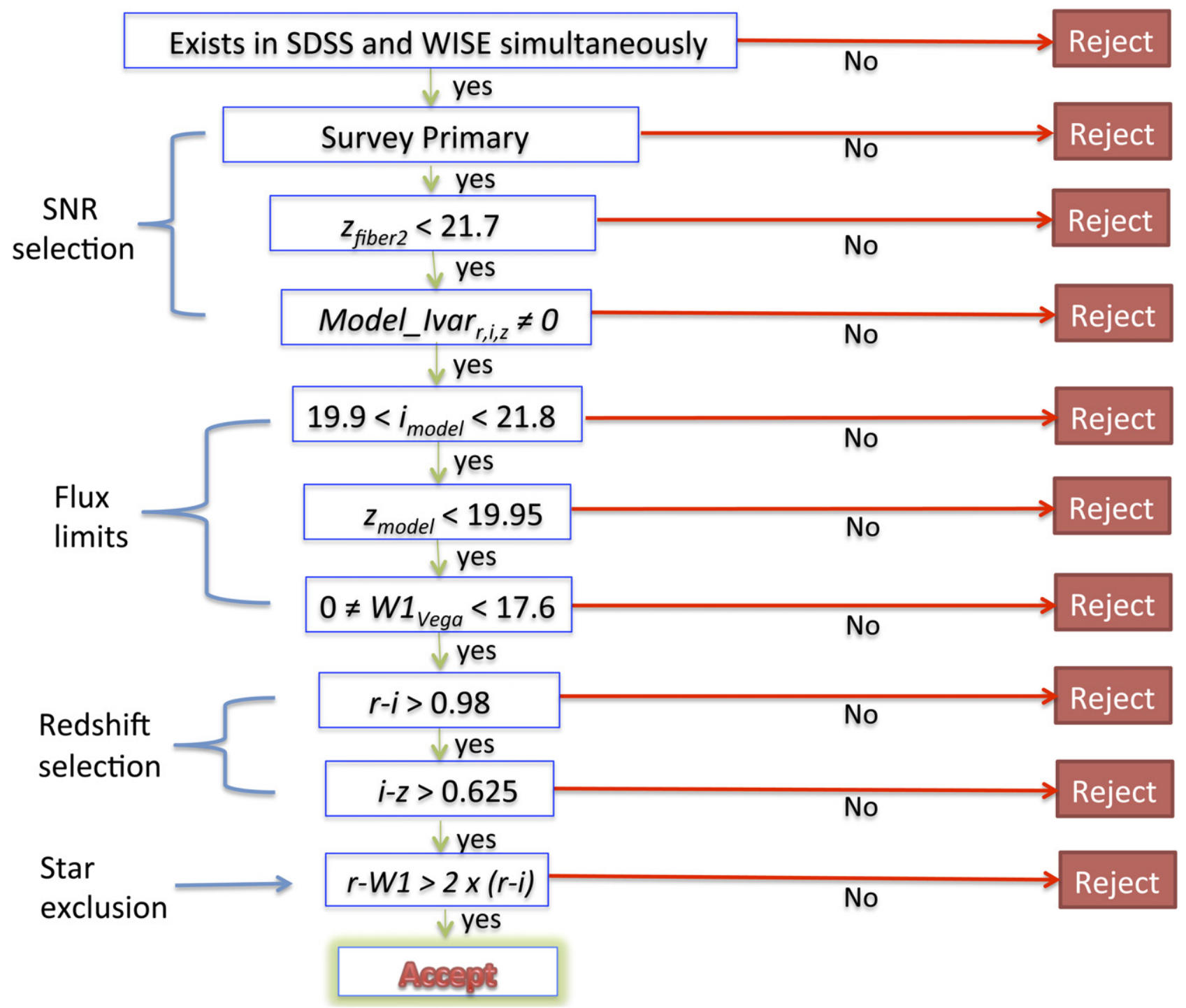

Figure 3. Schematic flow chart for the eBOSS LRG target selection algorithm. All quantities are corrected for Galactic extinction. See the text for a full description of all of the quantities shown in this figure.

conducted a visual inspection of a subset of eBOSS spectra, employing the idlspec $2 \mathrm{~d}$ package for this purpose. ${ }^{26}$

Specifically, we present results based on 2557 LRG candidates from eight plates that were visually inspected to assess the quality of spectra and robustness of redshift measurements by a team of eBOSS members. Each plate was inspected by multiple individuals to cross-check the results. Visual inspectors selected what they believed to be the best estimate of the correct redshift for each spectrum, as well as assessing the security of that redshift according to a simple four level confidence metric, $z_{-}$conf (confidence of inspector in the measured redshift). Targets are assigned $z_{-}$conf values of $0-3$, with 2 and 3 corresponding to measurements which were believed to be robust. A value $z_{\text {conf }}=1$ denotes a spectrum that is ambiguously classified, i.e., where more than one of the chisquared minima correspond to models which are a possible fit, while $z_{-}$conf $=0$ is used for objects where it is not possible to classify the objects and establish their redshift. These objects

$\overline{26}$ http://www.sdss3.org/dr8/software/products.php are considered unreliable and not used in the calculations of redshift distributions or related quantities.

In the remainder of this section, we briefly present the expected basic characteristics of the eBOSS LRG sample (e.g., its redshift distribution, spectral quality, and redshift success) derived from this sample with visual inspections. We also test the efficiency of our target selection algorithm against the science requirements for the eBOSS LRG sample as described in Section 2.

Two redshift distributions are presented in Table 1. The more conservative estimate (the one with a higher rate of "poor spectra") assumes that only objects given z_conf $>1$ have been assigned a correct redshift. The less conservative estimate includes all objects with $z_{-}$conf $>0$; this is a relevant scenario, since it is likely that a great majority of $z_{-} \operatorname{con} f=1$ redshifts are correct, but will inevitably include at least some incorrect redshifts. It is likely that the true distribution lies between these two bounds. It is expected that pipeline improvements now underway will enable at least some redshifts currently assigned $z_{-}$conf $<2$ to be recovered automatically in the future. 
Table 1

Redshift Distribution of eBOSS LRGs, Based upon Results for a Sample of 2557 Visually Inspected Spectra

\begin{tabular}{lcc}
\hline \hline & $\begin{array}{c}\text { LRGs } \\
\text { z_conf }>0\end{array}$ & $\begin{array}{c}\text { LRGs } \\
\text { z_conf }>1\end{array}$ \\
\hline Poor spectra & 4.0 & 6.7 \\
Stellar & 5.3 & 5.3 \\
Galaxy & $\mathrm{N} / \mathrm{A}$ & $\mathrm{N} / \mathrm{A}$ \\
$0.0<z<0.5$ & 0.6 & 0.6 \\
$0.5<z<0.6$ & 6.2 & 5.9 \\
$0.6<z<0.7$ & $\mathbf{1 5 . 2}$ & $\mathbf{1 4 . 8}$ \\
$0.7<z<0.8$ & $\mathbf{1 5 . 3}$ & $\mathbf{1 4 . 7}$ \\
$0.8<z<0.9$ & $\mathbf{9 . 4}$ & $\mathbf{8 . 7}$ \\
$0.9<z<1.0$ & $\mathbf{3 . 2}$ & $\mathbf{2 . 7}$ \\
$1.0<z<1.2$ & 0.6 & 0.5 \\
\hline Targets & 60 & 60 \\
Total Tracers & 43.1 & 41.0 \\
\hline
\end{tabular}

Note. The surface densities are presented in units of $\mathrm{deg}^{-2}$, normalizing to the total surface density of the parent sample for these spectra. Entries highlighted in bold font denote the subset of the sample that lies in the redshift range used to assess the high-level science requirements for the LRG sample.

As can be seen in the Table 1, even with the conservative scenario ( $z$ _con $f>1$ ), the SDSS spectral pipeline generates a secure redshift solution for $\sim 89 \%$ of the LRG candidates visually inspected. However, the fit determined to be correct via visual inspection sometimes does not correspond to the minimum chi-squared solution from the pipeline, but rather an alternative chi-squared minimum. ${ }^{27}$ Pipeline improvements now underway (which include both improved two-dimensional extractions and reductions in the freedom of template+polynomial fitting) are expected to improve the automated redshiftfinding, so this figure should be a floor to the actual performance of eBOSS LRGs.

The remaining $\sim 11 \%-12 \%$ of the LRG targets without a secure redshift determination typically have spectra with low $\mathrm{S} / \mathrm{N}$. An additional $\sim 9 \%$ of the LRG targets are found to be stars. These two factors (low $\mathrm{S} / \mathrm{N}$ and stellar contaminants) in combination, make it impossible for this sample to meet the eBOSS-only LRG requirement that that $80 \%$ of all targets be LRGs within the range $0.6 \lesssim z \lesssim 1.0$, even before the redshift distribution of the galaxies is considered. In the end, 68\%-72\% of all LRG targets are in fact galaxies with definitive redshift measurements that lie in the desired regime. For detailed discussion of the pipeline results, visual inspections, templates, and sources of redshift failures, see Dawson et al. (2016).

In Figure 4, we present the overall redshift distribution $(N(z))$ of the visually inspected eBOSS LRGs. Although we fail to meet the requirement of $80 \%$ efficiency at targeting $0.6<z<1.0$ LRGs, our target selection algorithm still exceeds the median redshift requirement, which is calculated only for actual galaxies (and hence includes only non-stellar targets with robust redshift measurements). In Figure 5, we show examples of LRG spectra across the redshift range of interest for eBOSS. There is an excellent match between the measured spectral energy distributions and the templates, confirming the robustness of these redshift measurements. The eBOSS LRG sample can be augmented with $z>0.6$ BOSS CMASS LRGs to meet our requirements on the total number of

${ }^{27}$ The SDSS pipeline generates a set of possible fits; cf. Bolton et al. (2012).

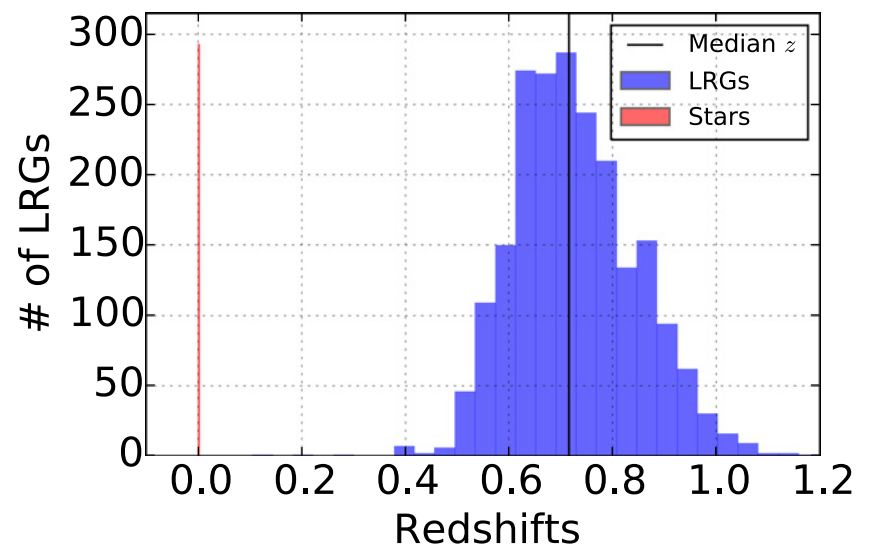

Figure 4. Redshift histogram of 2119 visually inspected LRGs (blue bar) observed with eBOSS. The median redshift of confirmed galaxies is 0.712 (black line), with $9 \%$ stellar contamination (red bar). We use only objects with secure redshifts $\left(z_{-}\right.$conf $\left.>1\right)$ here.

LRG redshifts within the range $0.6<z<1.0$; as a result, we still expect to achieve a $1 \%$ measurement of the LRG BAO scale at $z \sim 0.7$, even though the LRG sample falls short of its requirements.

\section{TESTS OF HOMOGENEITY AND IMPLICATIONS FOR LARGE-SCALE CLUSTERING MEASUREMENTS}

As discussed in Section 2.1, we require that the target sample be highly uniform to prevent non-cosmological signals from contaminating clustering measurements. Exploring systematics that can affect the inferred clustering of targets is often considered only when survey data are used for science analyses. We instead have investigated these issues while exploring target selection methods, enabling more informed decisions regarding survey strategy. For instance, foreknowledge of which areas of the survey may pose problems for controlling clustering measurements potentially allows the survey footprint to be shifted.

We assess the uniformity of the target sample by comparing the observed density of targets to maps of local imaging conditions and Galactic structure. We apply a regression analysis of surface density against a broad set of tracers of potential systematics; the intention is similar to, e.g., Scranton et al. (2002), Ross et al. (2011), Ho et al. (2012), Leistedt et al. (2013), Giannantonio et al. (2014), but unlike those works, we simultaneously fit for the impact of a wide variety of systematics rather than correlating against one at a time. This has the advantage of producing a model of systematic-affected density that will provide accurate predictions for the combined effects of all the systematics considered, even if the input systematic maps are covariant with each other (as, for instance, stellar density and dust extinction must inevitably be).

We focus on systematics associated with imaging data characteristics or with known astrophysical effects such as dust extinction and stellar density. Using the results of the regression analysis (described below) we assemble maps of the observed density and the predicted density. We identify regions within our footprint where the total span of target density fluctuation is less than $15 \%$, and consider the portion of sky with larger variations to be contaminated at an unacceptable level; this criterion is based on prior experience with the level of systematics that may be corrected reliably in BOSS Ross et al. (2011). We note that fluctuations in density within 

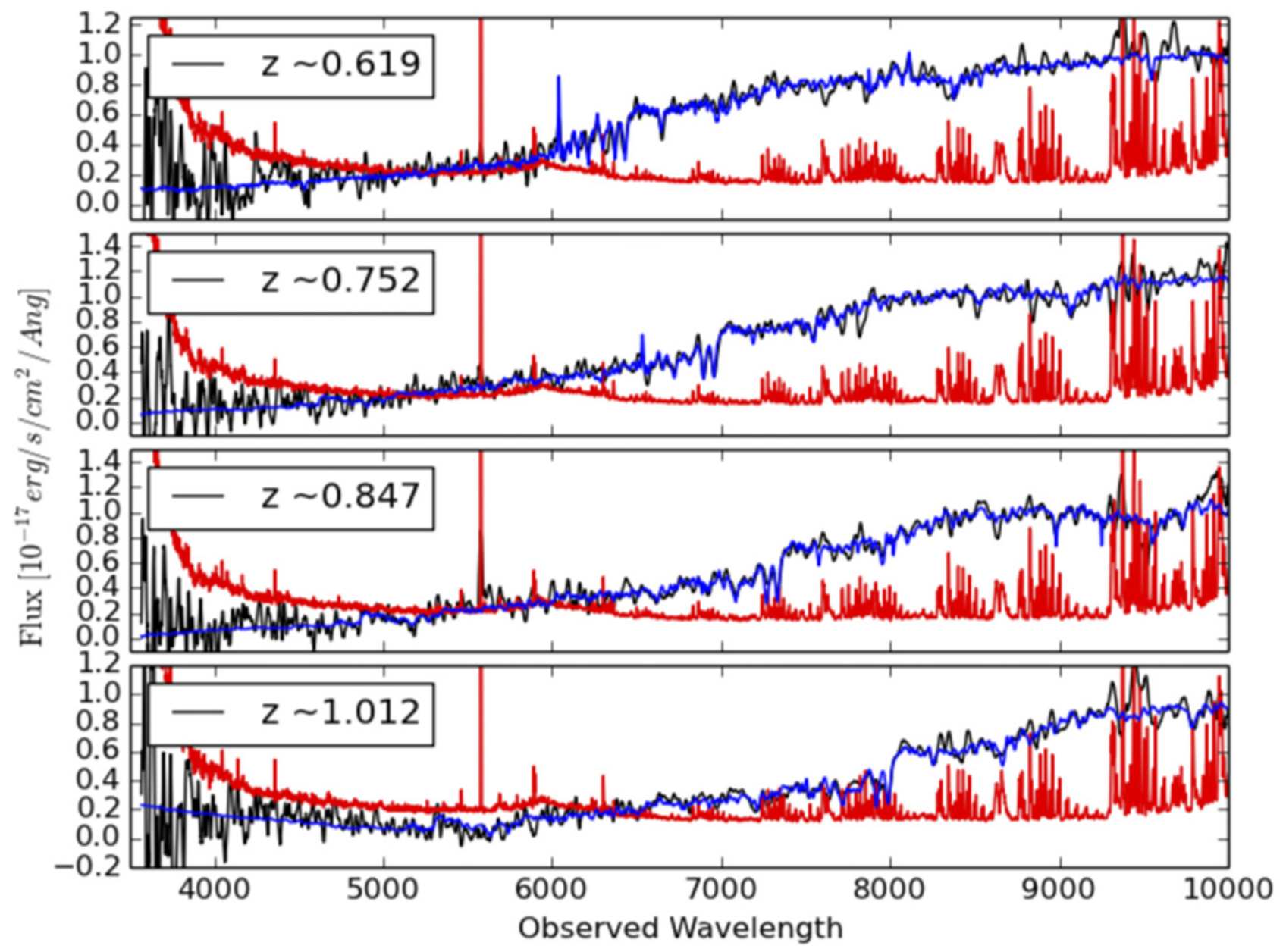

Figure 5. Representative spectra of galaxies from the eBOSS LRG sample, smoothed with a 21 pixel boxcar kernel. Shown are four LRGs covering the entire redshift regime of $0.6 \lesssim z \lesssim 1$. Flux errors are plotted in red while the template model fits are in blue. Black curves depict the observed spectra.

the final $0.6<z<1.0$ LRG catalog are likely to be smaller than this, as once spectra are obtained, stars and redshift outliers can be removed; such objects are naturally expected to be less homogeneous over the SDSS survey area than the true LRGs.

\subsection{Homogeneity of eBOSS LRG Targets}

To begin, we identify a broad set of imaging parameters that could affect eBOSS target selection.

1. W1 covmedian: the median number of single-exposure frames per pixel in the WISE W1-band.

2. moon_lev: the fraction of frames that were contaminated with scattered moon light in the WISE W1-band.

3. W1median: the median of accumulated flux per pixel in the WISE W1-band measured in units of $D N$ (data number). ${ }^{28}$

4. Galactic latitude: used as a proxy for stellar contamination.

5. Galactic extinction: we use $r$-band extinction, as given by SFD.

\footnotetext{
${ }^{28}$ The accumulated photons in each pixel are represented by a number in units of $D N$.
}

6. FWHM in the SDSS z-band: we use FWHM as an estimate of the "seeing" or imaging quality for the SDSS imaging.

7. SKYFLUX in the SDSS $z$-band: the background sky level affects the detection of faint objects is more difficult in the brighter regions of the sky.

We create maps of the WISE systematics over the entire SDSS footprint using the metadata tables associated with the Atlas images and source tables provided by WISE survey team; W1covmedian, W1median, and moon_lev are all quantities in these tables. ${ }^{29} \mathrm{We}$ use the seeing and the sky background in the $z$-band since the eBOSS LRG selection algorithm is flux-limited in that bandpass filter. Due to the scan strategy of SDSS, the seeing and sky background in other SDSS bands should correlate strongly with this quantity, making the use of multiple filters' quantities redundant.

Next, we break the sky up into equal-area pixels of $0.36 \mathrm{deg}^{2}$ and weight all pixels equally. The observed density, $S D_{\text {obs }}$, in each pixel can be expressed as a combination of a mean level, the impact of all of the systematics, and random noise:

$$
S D_{\mathrm{obs}}=S_{0}+\sum_{i=1}^{7} S_{i} \times x_{i}+\epsilon,
$$

\footnotetext{
${ }^{29}$ http://wise2.ipac.caltech.edu/docs/release/allsky/expsup/sec2_4f.html
} 
where $S_{0}$ is the constant term representing the mean density of objects in each pixel, $S_{i}$ are the coefficients for the values of each individual source of potential systematics fluctuations in that pixel $\left(x_{i}\right)$, and $\epsilon$ represents the combined effect of Poisson noise (or shot noise) and sample/cosmic variance in that pixel. For larger pixels such that the mean pixel target density is $\sim 15$ or more, the Poisson noise can be approximated as a Gaussian. Under these conditions, multi-linear regression provides an effective means of determining the unknown coefficients, $S_{0}$ and $S_{i}$. We derive a best-fit model based on minimizing the value of reduced- $\chi^{2}$ ( $\chi^{2}$ per degree of freedom). We have explored larger or smaller pixelizations and find that our results are unchanged.

The coefficients obtained from this multi-linear regression are then used in combination with the maps of potential systematics to predict the target density across the whole footprint, producing a statistic that we will refer to as the predicted surface density (PSD). We also define a residual surface density, or Residual_SD, for any particular systematic as the difference between $S D_{\text {obs }}$ and the Reduced_PSD (which is calculated by omitting the $j$ th systematic term in calculating the PSD). This quantity should be linear in systematic $j$ with a slope corresponding to $S_{j}$ if our linear regression model is appropriate to the problem. To summarize our formalism:

$$
\begin{gathered}
\mathrm{PSD}=S_{0}+\sum_{i=1}^{n} S_{i} \times x_{i}, \\
\text { Reduced_PSD} D_{j}=\mathrm{PSD}-S_{j} \times x_{j}, \text { and } \\
\text { Residual_SD }=\mathrm{SD}_{\mathrm{obs}}-\left(\mathrm{PSD}-S_{j} \times x_{j}\right),
\end{gathered}
$$

where the $j$ index indicates a single systematic of interest.

\subsection{PSD for eBOSS LRG Targets}

The PSD is highly useful for testing the uniformity of the target sample across the whole footprint, enabling comparisons to survey requirements. We find that the effects of systematics produce significantly different best-fit models (in terms of both the mean density and the coefficients for each systematic) in the areas of SDSS imaging around the NGC and the SGC. However, for both the regions considered independently, multilinear regression provides an acceptable best-fit model. Hence we analyze these regions separately.

The resulting regression fits are shown in Figure 6. In these plots, we plot the Residual_SD for each individual systematic which was been left out in calculating Residual_SD $D_{j}$. The data points plotted are averages over 4000 sky-pixels in the NGC or 2000 sky-pixels in the SGC; the error bars represent the standard error on the mean for each point. The straight lines represent the prediction from the regression model for the impact of the systematic indicated on the $x$-axis, $x_{j}$ (cf. Equation (13)); i.e., we plot $y=S_{j} \times x_{j}$.

\subsection{Analysis of Regression Results}

Our regression analysis allows us to determine what fraction of the survey footprint satisfies the requirement of less than $15 \%$ total variation in target density (point 6 in Section 2.2). This $15 \%$ window is not necessarily symmetric around the mean, so we fix its limits such that the footprint area satisfying the requirement is maximized. The windows containing regions with PSD variation $<15 \%$ are overplotted on the histograms of predicted density in Figure 7 . In the NGC, $\sim 97 \%$ of the imaging area meets the eBOSS survey requirements for homogeneity. However, in the SGC, only $\sim 82 \%$ of the area meets these requirements. At worst, these fluctuations will require that $8 \%$ of the total $7500 \mathrm{deg}^{2}$ eBOSS area is masked. However, these fluctuations may be reduced once spectroscopic redshifts are obtained; we will perform a similar analysis on the final spectroscopic sample in later work.

Differences in the observed number density between the NGC and SGC were found for the BOSS CMASS and LOWZ samples, and were analyzed in depth by Ross et al. (2012). These differences matched the photometric offsets between the two regions determined by Schlafly \& Finkbeiner (2011). These offsets have been incorporated into the re-calibrated photometry used for eBOSS; any difference in target density between the regions is therefore due to still-unknown differences between the two regions. This issue will require further investigation in future eBOSS studies.

Based on the regression model, we can assess which systematics are most strongly affecting target selection. We find that all of the potential WISE imaging systematics have relatively weak effects on the density of selected targets. This can be seen from the flatness of Residual_SD for these parameters in Figure 6. The most significant effects are associated with dust extinction, stellar contamination, and the SDSS sky background level, as seen from the steep slopes in Figure 6. It is unclear whether dust or stellar contamination is more fundamentally responsible for variations in density, since the two correlate with each other strongly. Given the variation in coefficients, it is likely that the same phenomenon is being ascribed more to dust in the NGC and to Galactic latitude in the SGC, and those differences in coefficient are not truly significant. Fortunately, the regression model will still predict the correct density from covariant variables such as these, regardless of which covariate is actually responsible.

We depict the observed surface density, the PSD, and the mask of the survey across the whole footprint of SDSS in Figure 8.

\subsection{Impact of Zero-point Variations}

We next assess the expected level of variation in target density due to errors in zero-point calibrations, which can then be compared to the targeting requirements. We investigate this by determining the fractional derivative in the number of targets selected $(N)$ as we shift all magnitudes in a given band ( $m$ ) by a constant amount-i.e., we calculate $\frac{1}{N} d N / d m$ - and then assess what impact this sensitivity has on target density. We find that zero-point errors of $0.01 \mathrm{mag}$ in the $r-, i-, z^{-}$, and $W 1$-bands cause fractional changes of $2.26 \%, 2.5 \%, 6.24 \%$, and $0.6 \%$, respectively, in the target density of the LRG sample. Finkbeiner et al. (2014) estimate that the $1 \sigma$ zero-point uncertainties $\left(\sigma_{z p}\right)$ after recalibration of SDSS are 7, 7, and 8 millimagnitudes in the SDSS $r$-, $i$-, and $z$-bands respectively, while WISE calibration uncertainties in the W1-band are approximately 0.016 mag (Jarrett et al. 2011).

Assuming that zero-point errors will be Gaussian-distributed, $95 \%$ of all points on the sky will be within $\pm 2 \sigma$ of the mean zero point. Hence, the total fractional variation in density over that area will be $\frac{4}{N} \times|\Delta N / \Delta m| \times \sigma_{z p}$. We present the results of this calculation in Table 2 and Figure 9. For all bands but $z$, the impact of zero-point variations on the density of LRG targets will be minimal. However, the estimated level of $z$-band 
NGC

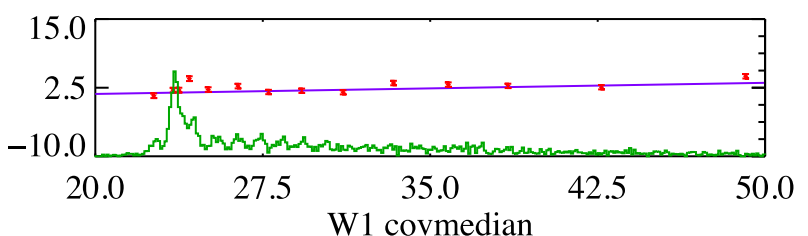

$\mathrm{W} 1$ covmedian
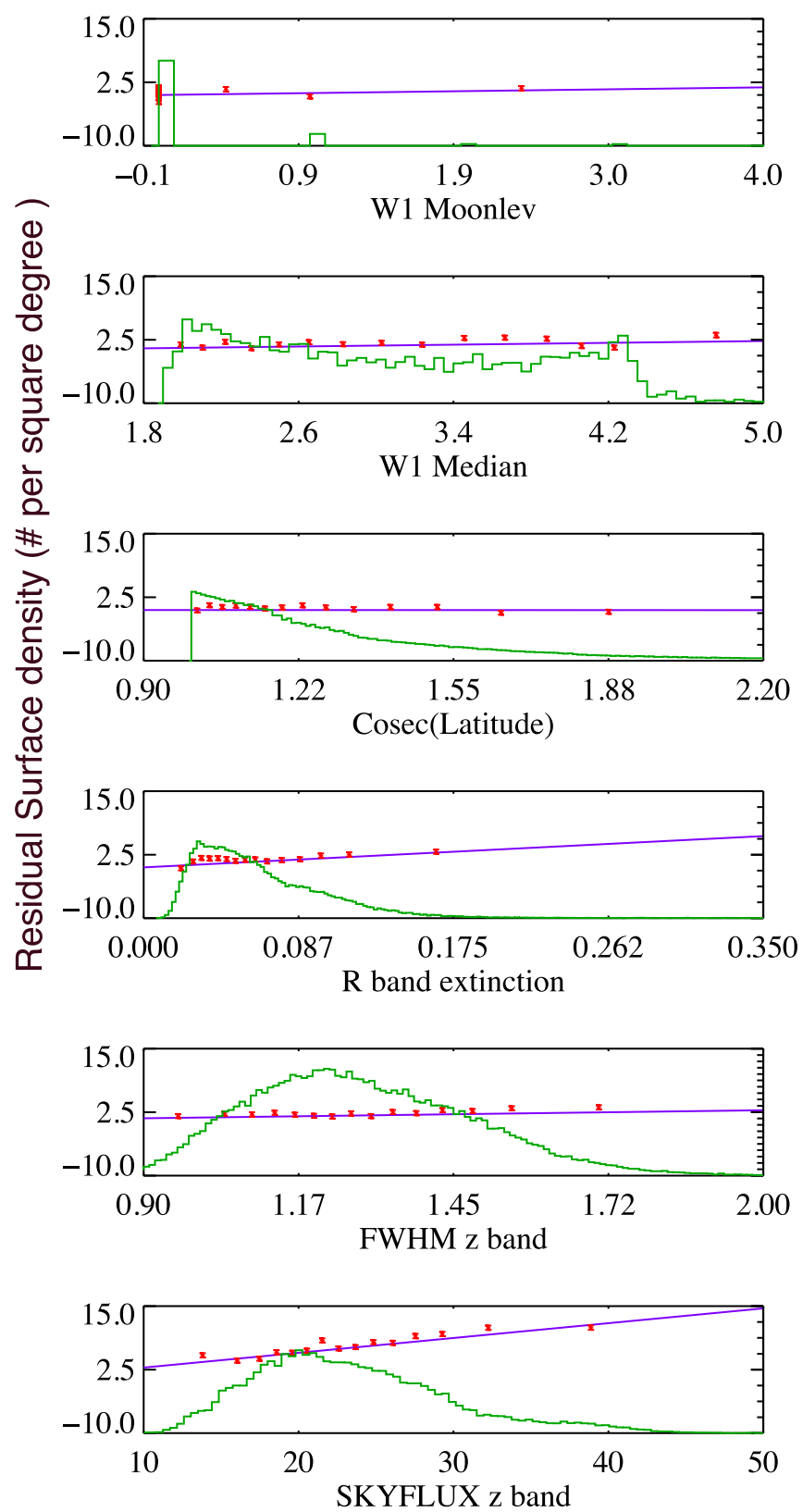

SGC
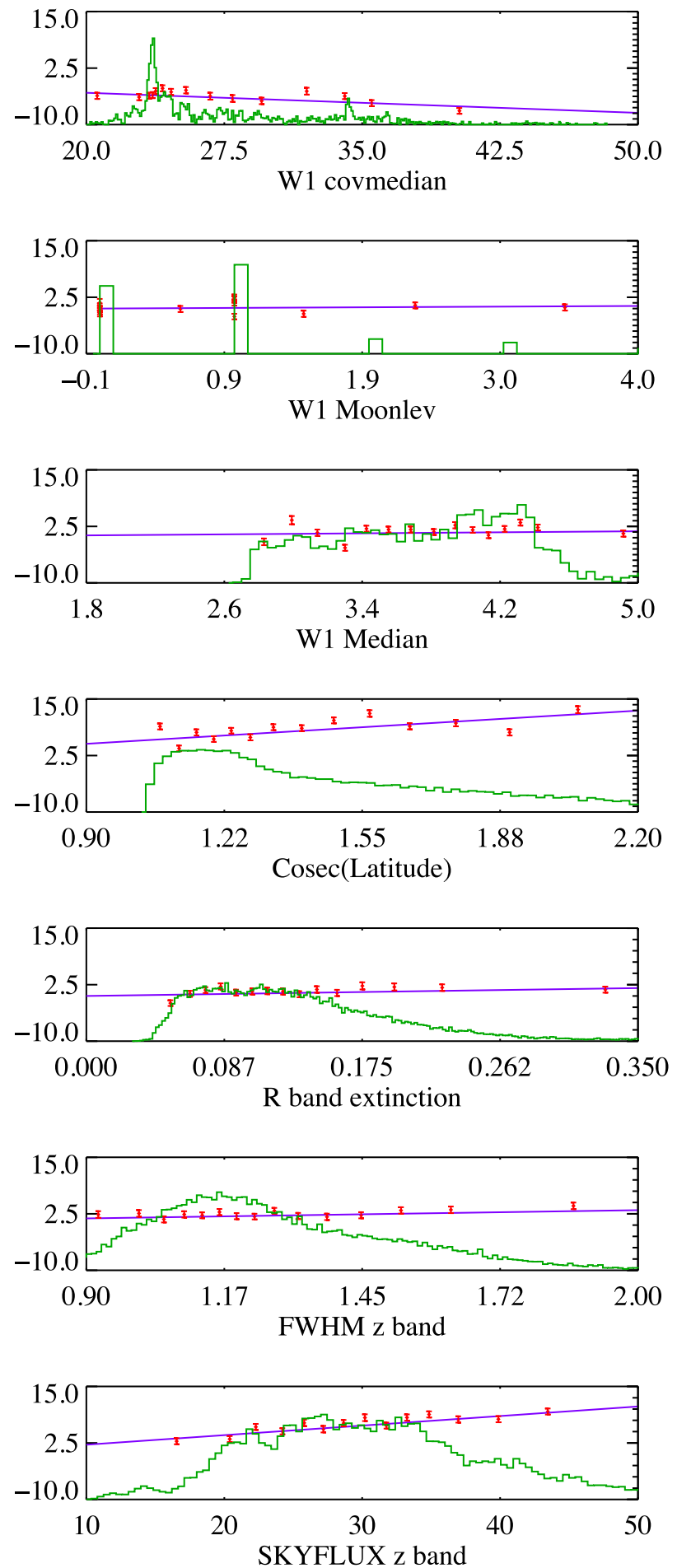

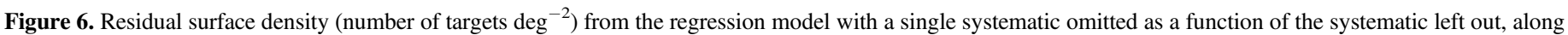

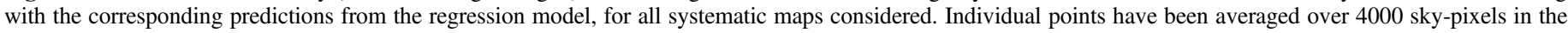

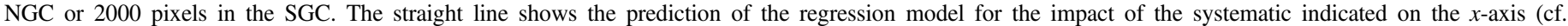

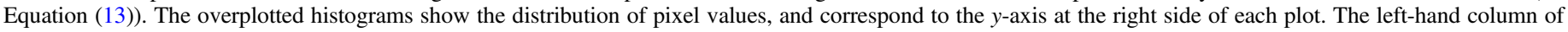
plots are for pixels in the NGC, while the right-hand column of plots are for the SGC. A linear model appears to be appropriate for all systematics considered.

zero-point uncertainty is sufficiently large that more than $13 \%$ of the eBOSS area will go beyond the $15 \%$ target density variation requirement. The impact of this variation and strategies for mitigating it will be explored in future eBOSS papers.

\section{CONCLUSIONS}

The LRG component of SDSS-IV/eBOSS will obtain spectroscopy of a sample of over 375,000 potential intrinsically luminous early-type galaxies at $z>0.6$. Based on the initial set of eBOSS data, we find that the efficiency of this selection for 


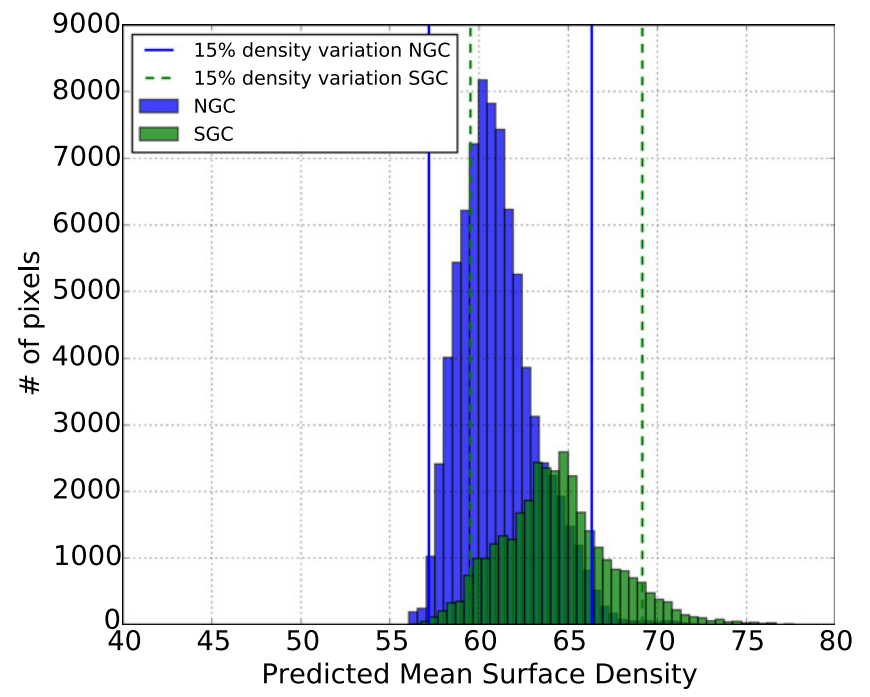

Figure 7. Histogram of the surface density predicted by the regression models described in Section 7.1. The blue bars represent the NGC, with solid blue lines depicting the $15 \%$ window within which samples are expected to be sufficiently homogeneous for robust large-scale structure measurements. Similarly, the green bars represent the density in the SGC, with dotted green lines depicting the $15 \%$ window. We find that $~ 97 \%$ of the NGC footprint with SDSS imaging meets the homogeneity requirements of eBOSS (see Section 2.2). However, in the SGC, only $\sim 82 \%$ of the possible eBOSS footprint meets these requirements.

selecting $0.6<z<1.0$ spectroscopically confirmed LRGs with secure redshift measurements is $\sim 68 \%-72 \%$. Although this is lower than the required level for eBOSS to achieve its science goals in isolation, once augmented with BOSS LRGs the required galaxy density should be attained. One reason the success rate does not approach $80 \%$ is that $9 \%$ of LRG targets prove to be stars, a result of noisy SDSS photometry. The sample is flux-limited to keep the selection algorithm robust, as well as to maintain a sufficient $\mathrm{S} / \mathrm{N}$ to enable the resulting LRG spectra to provide secure redshift measurements. The LRG sample is uniform and homogenous over $\sim 92 \%$ of the BOSS survey footprint, showing little or no dependence on imaging systematics and flux calibrations. The remaining $\sim 8 \%$ of the footprint will have to be assessed carefully for systematic effects before being included in cosmology measurement if they fall within eBOSS footprint.

The primary science drivers of the eBOSS LRG sample are to study the large-scale structure of the universe out to $z \sim 1$. With careful control of incompletenesses and selection effects, the eBOSS LRG algorithm will also provide a large sample for galaxy evolution studies of giant elliptical galaxies. The SDSS-IV/eBOSS LRGs will cover a volume either not probed, or not probed at high density, by SDSS-III/BOSS, and will allow both $\mathrm{BAO}$ and RSD measurements with a highly uniform set of luminous early-type galaxies. The SDSS-IV/eBOSS LRG sample will provide a powerful extension of SDSS-III/ BOSS for the study of structure and galaxy evolution at high redshifts.

This work was supported by an Early Career Grant from the U.S. Department of Energy Office of Science. This paper represents an effort by both the SDSS-III and SDSS-IV collaborations. Funding for SDSS-III was provided by the Alfred P. Sloan Foundation, the Participating Institutions, the
National Science Foundation, and the U.S. Department of Energy Office of Science.

This paper includes targets derived from the images of the Wide-Field Infrared Survey Explorer, which is a joint project of the University of California, Los Angeles, and the Jet Propulsion Laboratory/California Institute of Technology, funded by the National Aeronautics and Space Administration.

Funding for the Sloan Digital Sky Survey IV has been provided by the Alfred P. Sloan Foundation, the U.S. Department of Energy Office of Science, and the Participating Institutions. SDSS-IV acknowledges support and resources from the Center for High-Performance Computing at the University of Utah. The SDSS web site is www.sdss.org. SDSS-IV is managed by the Astrophysical Research Consortium for the Participating Institutions of the SDSS Collaboration including the Brazilian Participation Group, the Carnegie Institution for Science, Carnegie Mellon University, the Chilean Participation Group, the French Participation Group, Harvard-Smithsonian Center for Astrophysics, Instituto de Astrofísica de Canarias, The Johns Hopkins University, Kavli Institute for the Physics and Mathematics of the universe (IPMU)/University of Tokyo, Lawrence Berkeley National Laboratory, Leibniz Institut für Astrophysik Potsdam (AIP), Max-Planck-Institut für Astronomie (MPIA Heidelberg), MaxPlanck-Institut für Astrophysik (MPA Garching), Max-PlanckInstitut für Extraterrestrische Physik (MPE), National Astronomical Observatory of China, New Mexico State University, New York University, University of Notre Dame, Observatário Nacional/MCTI, The Ohio State University, Pennsylvania State University, Shanghai Astronomical Observatory, United Kingdom Participation Group, Universidad Nacional Autónoma de México, University of Arizona, University of Colorado Boulder, University of Portsmouth, University of Utah, University of Virginia, University of Washington, University of Wisconsin, Vanderbilt University, and Yale University.

\section{APPENDIX A}

\section{A.1. $L R G \_I D R O P$}

Objects with LRG-like colors which are too faint for detection in the $i$-band but still have a robust detection in the $z$-band can be targeted via a different color-cut. The $r$-band photometry for these objects becomes quite noisy and hence it is not used in selection. Instead, we can use a similar selection in a different optical-IR color-color space:

$$
\begin{gathered}
i_{\text {Model }}>21.8, \\
z_{\text {Model }} \leqslant 19.5, \\
i-z>0.7, \\
i-W 1>2.143 \times(i-z)-2.0 .
\end{gathered}
$$

Equations (19) and (20) represent an analogous color selection to Equations (10) and (11), but using the $i$ - and $z$-bands instead of $r$ and $i$. Equation (18) ensures that the objects are welldetected in the $z$-band despite having a noisy detection (if any) in bluer bands. This selection contributes a few targets, 200 over the entire footprint, which are expected to be at higher redshifts than the standard eBOSS LRG sample. 

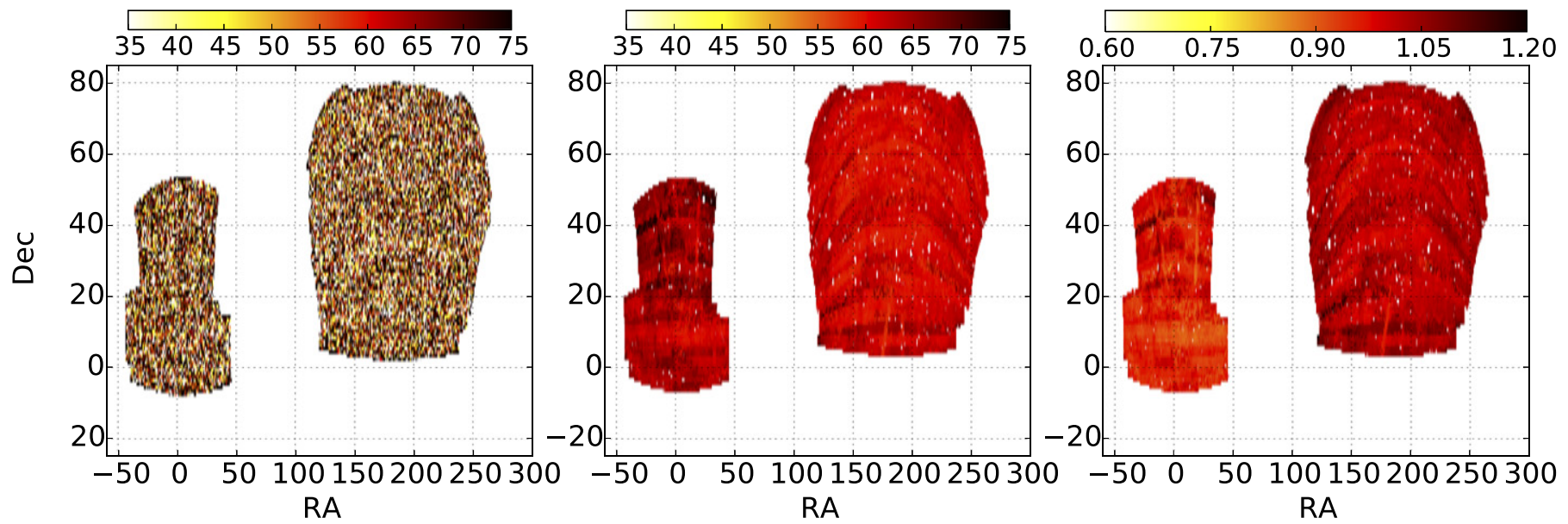

Figure 8. Observed surface density map of eBOSS LRGs over the area of the SDSS imaging footprint used to derive targets for the BOSS survey. eBOSS will target more than $\sim 375,000$ LRGs over a $\sim 7500 \mathrm{deg}^{2}$ subset of this area, corresponding to a surface density of $\sim 50 \mathrm{deg}^{-2}$. The color scale in this panel is dominated by Poisson noise and sample/cosmic variance. The middle panel shows a similar plot based on the predicted density, while the third panel shows the regions which would be masked to reach eBOSS homogeneity requirements. Pixels shown with a shade that is fainter or darker than the typical are those which fail to meet the $15 \%$ target density variation requirement of Section 2.2.

Table 2

Summary of Variations in Target Density due to Errors in SDSS Imaging Zero Points

\begin{tabular}{lccc}
\hline \hline Bands & $\begin{array}{c}\text { Derivative of } \\
\text { Fractional Density } \\
\left|\frac{1}{N} \Delta N / \Delta m\right|\end{array}$ & $\begin{array}{c}\text { rms Zero-point } \\
\text { Error }\end{array}$ & $\begin{array}{c}95 \% \text { Range of Variation } \\
\text { in Fractional Density }\end{array}$ \\
& & $\left(\sigma_{z p}\right)$ & $7 \times|\Delta \bar{N} / \Delta \mathrm{Mag}| \times \sigma_{z p}$ \\
\hline SDSS $r$ & 2.26 & $7 \times 10^{-3}$ & 0.063 \\
SDSS $i$ & 2.5 & $8 \times 10^{-3}$ & 0.070 \\
SDSS $z$ & 6.24 & $16 \times 10^{-3}$ & 0.199 \\
WISE $W 1$ & 0.60 & 0.038
\end{tabular}

Note. The impact of zero-point uncertainties on the density of targets. The eBOSS LRG sample meets the requirement that density variations due to zero-point errors be less than $15 \%$ in the SDSS $r$ and $i$ bands, but fails to meet that criterion in the $z$-band with current calibrations.

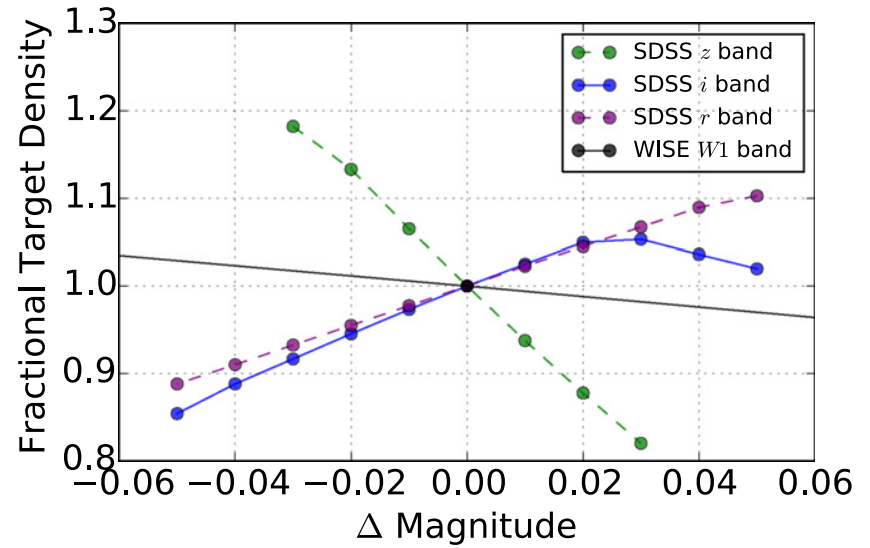

Figure 9. Change in target density as a function of an overall shift in all magnitudes in either the SDSS $r, i$, and $z$ band, or the WISE W1 band. Given the current level of zero-point uncertainty in SDSS and WISE photometry, the LRG target selection is only sensitive to the uncertainty in the zero point of the $z$-band.

\section{APPENDIX B}

\section{B.1. Results from a Large Pilot Survey, SEQUELS}

As mentioned in Section 4, the basic ideas underlying the eBOSS selection algorithm can be implemented in a variety of optical-IR color spaces. To determine the optimum selection algorithm between two candidate methods, we selected $\sim 70,000$ LRGs over an area of $\sim 700 \mathrm{deg}^{2}$ with $120^{\circ} .0<\alpha<210^{\circ} .0$ and $45^{\circ} .0<\delta<60^{\circ} .0$. These LRGs were selected by algorithms utilizing two different optical-IR color spaces, and were used to test our selection efficiency and redshift success. The parameters of the selection algorithms were tuned such that one obtains a target density of $\sim 60 \mathrm{deg}^{-2}$ from each one. In the following sub-sections, we explain the two selection algorithms with their commonalities and major differences.

\section{B.2. Common Cuts for SEQUELS LRG Samples}

First, we require that the RESOLVE_STATUS bit corresponding to SURVEY PRIMARY is nonzero in order to remove duplicate objects. We also require the photometric flag have the CALIB_STATUS bit set for all of the $r, i$, and $z$ bands used for photometric color determinations. In addition, the following flux limits are applied over the entire sample:

$$
\begin{gathered}
z_{\text {Fiber2 }} \leqslant 21.7, \text { and } \\
i_{\text {Model }} \geqslant 19.9 .
\end{gathered}
$$

\section{B.2.1. $\mathrm{r} / \mathrm{i} / \mathrm{z} /$ WISE LRG Selection}

In the first selection, we identify LRGs using $r-W 1, r-i$, and $i-z$ color. This selection algorithm is very similar to the 
selection described in Section 5, differing only due to changes in flux limits to improve completeness. In addition to the common cuts described above, we apply the following selection criteria:

$$
\begin{gathered}
z_{\text {Model }} \leqslant 19.95, \\
r-i>0.98, \\
r-W 1>2.0 \times(r-i), \text { and } \\
i-z>0.625,
\end{gathered}
$$

where all variables have the same meanings as in Section 5.2. These equations and their relevance have been explained previously in Section 5.

\section{B.2.2. $\mathrm{i} / \mathrm{z} /$ WISE LRGs}

The second selection is implemented exclusively in $i-W 1$ and $i-z$ optical-IR color-color space, eliminating any use of the $r$ band. This selection algorithm is similar to the one explained in Appendix A, differing primarily in its flux limits, which have been tuned to produce the same target density as the $r / i / z / W I S E$ selection. In addition to the common cuts, we apply the following selection criteria:

$$
\begin{gathered}
z_{\text {Model }} \leqslant 19.5, \\
i-z>0.7, \text { and } \\
i-W 1>2.143 \times(i-z)-2.0 .
\end{gathered}
$$

The equations and their relevance are the same as explained previously in LRG_IDROP (Appendix A).

\section{B.3. Details of the SEQUELS Survey}

SEQUELS was conceived as a precursor of eBOSS enabling us to test the reliability and efficiency of our selection algorithms while simultaneously producing data that could be combined with the full eBOSS data set to constrained cosmology. It provided a sufficiently large data set to enable robust tests of selection algorithms. It was also critical in testing and demonstrating our ability to meet eBOSS requirements via these selection algorithms. We applied both of the selection algorithms explained in the section Appendix B.2 in parallel over the entire SDSS footprint. The final SEQUELS LRG sample consisted of the objects selected by either or both of the selection algorithms explained above.

\section{B.3.1. Targeting Bits}

In order to identify LRGs selected via different algorithms, we assign them different values of the eBOSS_TARGETO tag. For LRGs selected in $i / z /$ WISE color space, eBOSS_TARGETO is set bit-wise to $1 .{ }^{30}$ For LRGs selected via $r / i / z / W I S E$ selection, eBOSS_TARGETO is set bit-wise to 2. LRGs which pass both of the selection criteria have both bits set.

\section{B.3.2. Overall Characteristics of SEQUELS LRGs}

The two classes of LRGs, i.e., $r / i / z /$ WISE selected and $i / z /$ WISE selected, were analyzed separately. We found that $\gtrsim 87 \%$ of spectra yielded secure redshift measurements. Redshift measurements are checked via visual inspection of the spectra. The remaining $13 \%$ were found to have small differences between the depths of the lowest chi-squared minima, and

$\overline{{ }^{30} \text { Bit } 0,1,2 \text { are used to indicate } 2^{0}=1,2^{1}}=2$, and $2^{2}=4$, respectively.
Table 3

Summary of $r / i / z /$ WISE and $i / z /$ WISE in Comparison to Key eBOSS Requirements

\begin{tabular}{lccl}
\hline \hline Requirement & $r / i / z /$ WISE & $i / z /$ WISE & Summary \\
\hline \# of targets: & 450,000 & 450,000 & Easily achievable \\
$>375,000$ & $(\sim 60$ & $(\sim 60$ & \\
& $\begin{array}{c}\text { targets } \\
\left.\operatorname{deg}^{-2}\right)\end{array}$ & $\begin{array}{c}\text { targets } \\
\left.\operatorname{deg}^{-2}\right)\end{array}$ & \\
& 0.716 & 0.697 & $i / z /$ WISE failing \\
Median Redshift: & & & marginally \\
$>0.71$ & $\sim 71 \%$ & $\sim 64 \%$ & Both samples \\
$\begin{array}{l}\text { Fraction at } 0.6 \lesssim z \\
\quad \lesssim 1.0:\end{array}$ & & fail to meet \\
$>80 \%$ & & & \\
\hline
\end{tabular}

Note. The $r / i / z /$ WISE selection meets the basic median redshift requirement which is necessary to achieve our science goals. However, both algorithms fail to meet the redshift efficiency requirement. $r / i / z / W I S E$ selects more highredshift LRGs and hence was chosen as the preferred selection algorithm for eBOSS.

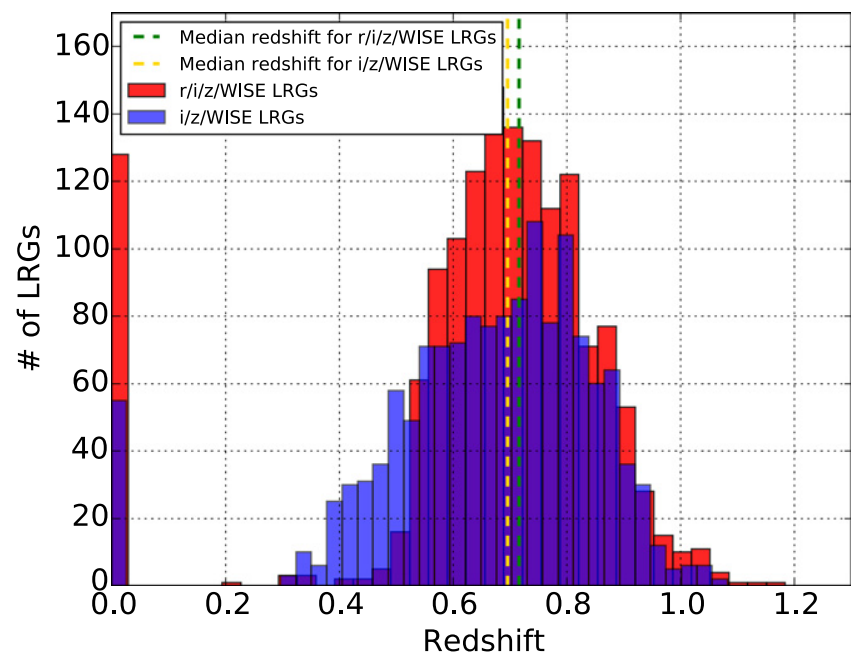

Figure 10. Redshift histogram of $\sim 1500$ visually inspected LRGs targeted by two different selection algorithms as part of SEQUELS. $r / i / z /$ WISE selects more LRGs at higher redshift. In contrast, $i / z / W I S E$ selects more LRGs at lower redshifts which are less useful for eBOSS. Hence, $r / i / z /$ WISE is the preferred choice for the eBOSS LRG sample.

hence were judged not to be reliable; this generally occurred due to low $\mathrm{S} / \mathrm{N}$ in the spectra. $8 \%$ of the total targets were both classified securely and found to be stars. These two factors (13\% of targets having no definitive redshift measurement and another $8 \%$ being stars) make it impossible to reach the required efficiency at targeting $0.6<z<1.0$ LRGs of $80 \%$. We meet the requirement set on the eBOSS median redshift using the $r / i / z /$ WISE algorithm, but not the $i / z /$ WISE algorithm. Among the objects which failed to yield a secure redshift measurement, most were noise-dominated. We tabulate the key results in Table 3.

In Figure 10, we present the redshift distributions, $N(z)$, of $r /$ $i / z /$ WISE and $i / z /$ WISE LRGs. We find that the $i / z /$ WISE selection algorithm selects a significantly higher fraction of fraction of galaxies at $0.4<z<0.5$ compared to the $r / i / z /$ WISE selection. This causes the median redshift and targeting efficiency to fall below our requirements, as seen also in Table 3. Overall, $r / i / z / W I S E$ was found to be more suitable for eBOSS. It gains greater efficiency by requiring targets to be red in both $r-i$ and $i-z$, providing a veto in cases where one 
color is affected by bad photometry. However, at redshifts $z \gtrsim$ 0.75 both of the candidate selection algorithms yielded similar results.

\section{B.4. Differences Between SEQUELS and eBOSS Targets}

Post SEQUELS, we made a few improvements in our target selection algorithm. These changes are expected to improve our secure redshift measurement rate by removing objects whose counterparts yielded extremely low $\mathrm{S} / \mathrm{N}$ spectra in SEQUELS. For eBOSS LRGs, we add two additional criteria to the SEQUELS $r / i / z / W I S E$ selection:

$$
\begin{gathered}
W 1_{\mathrm{AB}} \leqslant 20.299, \text { and } \\
i_{\text {Model }} \leqslant 21.8 .
\end{gathered}
$$

Equation (30) effectively requires a $5 \sigma$ detection in the first channel (W1) of WISE. In addition, we put a faint limit on $i_{\text {Modelflux }}$ through Equation (31); this was not applied in SEQUELS. These additional flux limits reduce the number of noise-dominated LRG spectra significantly when applied to the SEQUELS sample.

\section{REFERENCES}

Abazajian, K. N., Adelman-McCarthy, J. K., Agüeros, M. A., et al. 2009, ApJS, 182, 543

Aihara, H., Allende Prieto, C., An, D., et al. 2011, ApJS, 195, 26

Alam, S., Albareti, F. D., Allende Prieto, C., et al. 2015, ApJS, 219, 12

Beutler, F., Saito, S., Brownstein, J. R., et al. 2014a, MNRAS, 444, 3501

Beutler, F., Saito, S., Seo, H.-J., et al. 2014b, MNRAS, 443, 1065

Bolton, A. S., Schlegel, D. J., Aubourg, É., et al. 2012, AJ, 144, 144

Cannon, R., Drinkwater, M., Edge, A., et al. 2006, MNRAS, 372, 425

Comparat, J., Delubac, T., Jouvel, S., et al. 2015, arXiv:1509.05045

Dawson, K. S., Kneib, J.-P., Percival, W. J., et al. 2015, arXiv:1508.04473

Dawson, K. S., Kneib, J.-P., Percival, W. J., et al. 2016, AJ, 151, 44

Dawson, K. S., Schlegel, D. J., Ahn, C. P., et al. 2013, AJ, 145, 10

Eisenstein, D. J., Annis, J., Gunn, J. E., et al. 2001, AJ, 122, 2267

Eisenstein, D. J., Weinberg, D. H., Agol, E., et al. 2011, AJ, 142, 72

Eisenstein, D. J., Zehavi, I., Hogg, D. W., et al. 2005, ApJ, 633, 560
Finkbeiner, D. P., Schlafly, E. F., Schlegel, D. J., et al. 2016, ApJ, 822, 66 Fukugita, M., Ichikawa, T., Gunn, J. E., et al. 1996, AJ, 111, 1748

Giannantonio, T., Ross, A. J., Percival, W. J., et al. 2014, PhRvD, 89, 023511

Gunn, J. E., Carr, M., Rockosi, C., et al. 1998, AJ, 116, 3040

Gunn, J. E., Siegmund, W. A., Mannery, E. J., et al. 2006, AJ, 131, 2332

Gwyn, S. D. J. 2011, arXiv:1101.1084

Ho, S., Cuesta, A., Seo, H.-J., et al. 2012, ApJ, 761, 14

Ilbert, O., Salvato, M., Capak, P., et al. 2008, in ASP Conf. Ser. 399,

Panoramic Views of Galaxy Formation and Evolution, ed. T. Kodama,

T. Yamada, \& K. Aoki (San Francisco, CA: ASP), 169

Jarrett, T. H., Cohen, M., Masci, F., et al. 2011, ApJ, 735, 112

John, T. L. 1988, A\&A, 193, 189

Jones, D. 1968, ASPL, 10, 145

Kaiser, N., Burgett, W., Chambers, K., et al. 2010, Proc. SPIE, 7733, 0

Kauffmann, G., White, S. D. M., Heckman, T. M., et al. 2004, MNRAS, 353,713

Lang, D., Hogg, D. W., \& Schlegel, D. J. 2014, arXiv:1410.7397

Leistedt, B., Peiris, H. V., Mortlock, D. J., Benoit-Lévy, A., \& Pontzen, A. 2013, MNRAS, 435, 1857

Lin, Y.-T., \& Mohr, J. J. 2003, ApJ, 582, 574

Lupton, R. H., Gunn, J. E., \& Szalay, A. S. 1999, AJ, 118, 1406

Myers, A. D., Palanque-Delabrouille, N., Prakash, A., et al. 2015, ApJS, 221, 27

Oke, J. B., \& Gunn, J. E. 1983, ApJ, 266, 713

Padmanabhan, N., Schlegel, D. J., Finkbeiner, D. P., et al. 2008, ApJ, 674, 1217

Ade, P. A. R., Aghanim, N., et al. 2014, A\&A, 571, A16

Postman, M., \& Geller, M. J. 1984, ApJ, 281, 95

Postman, M., \& Lauer, T. R. 1995, ApJ, 440, 28

Prakash, A., Licquia, T. C., Newman, J. A., \& Rao, S. M. 2015, ApJ, 803, 105

Ross, A. J., Ho, S., Cuesta, A. J., et al. 2011, MNRAS, 417, 1350

Ross, A. J., Percival, W. J., Sánchez, A. G., et al. 2012, MNRAS, 424, 564

Ross, A. J., Samushia, L., Burden, A., et al. 2014, MNRAS, 437, 1109

Ross, N. P., Shanks, T., Cannon, R. D., et al. 2008, MNRAS, 387, 1323

Samushia, L., Reid, B. A., White, M., et al. 2014, MNRAS, 439, 3504

Schlafly, E. F., \& Finkbeiner, D. P. 2011, ApJ, 737, 103

Schlafly, E. F., Finkbeiner, D. P., Jurić, M., et al. 2012, ApJ, 756, 158

Schlegel, D. J., Finkbeiner, D. P., \& Davis, M. 1998, ApJ, 500, 525

Scranton, R., Johnston, D., Dodelson, S., et al. 2002, ApJ, 579, 48

Seo, H.-J., \& Eisenstein, D. J. 2003, ApJ, 598, 720

Smee, S. A., Gunn, J. E., Uomoto, A., et al. 2013, AJ, 146, 32

Stoughton, C., Lupton, R. H., Bernardi, M., et al. 2002, AJ, 123, 485

Wright, E. L., Eisenhardt, P. R. M., Mainzer, A. K., et al. 2010, AJ, 140, 1868

York, D. G., Adelman, J., Anderson, J. E., Jr., et al. 2000, AJ, 120, 1579 